\title{
An investigation of the melting process of RT-35 filled circular thermal energy storage system
}

https://doi.org/10.1515/phys-2018-0075

Received September 17, 2017; accepted July 4, 2018

\begin{abstract}
One of the main solutions to the issue of global warming and greenhouse gas emission caused by burning fossil fuels is storing energy in an efficient way. In this work, the detailed melting process of RT-35 as a phase change material (PCM) inside a cylindrical latent heat thermal energy storage (TES) system is investigated both numerically and experimentally. To achieve this aim, an experimental setup comprising of a transparent vertical cylindrical enclosure as a latent heat TES system, a constant temperature bath, and a temperature regulator is built. Moreover, a numerical model using COMSOL multiphysics is developed to simulate the melting process and provide a more detailed information on the flow and thermal fields. The model is able to provide the temperature and velocity fields, heat transfer behaviour, melting fraction, and the trend of solid-liquid interface at different time intervals. To validate the numerical model, a comparison between melting fraction and solid-liquid interfaces of the numerical model and experimental work is conducted which shows a good agreement between experimental and numerical results.
\end{abstract}

Keywords: Thermal energy storage (TES) systems; Phase change material (PCM); Solid-liquid interface; Rubitherm

PACS: 44.05.+e, 44.10.+i, 44.25.+f, 44.35.+c

\section{Introduction}

Nowadays, the increase in the energy consumption due to the growth of population and development of industries, environmental concerns due to global warming, depletion of fossil fuel sources and increase in the price of ex-

\footnotetext{
^Corresponding Author: Soroush Ebadi: School of Engineering, University of Guelph, Guelph, ON, Canada,

E-mail: sebadi@uoguelph.ca

Manar Al-Jethelah, Syeda Humaira Tasnim, Shohel Mahmud:

School of Engineering, University of Guelph, Guelph, ON, Canada
}

tracting them are the main concerns regarding energy supply. These economic and environmental concerns have enforced energy providers and researchers to look for an affordable solution to remedy this issue. Storing energy in an efficient way can play a significant role to reduce the burning of fossil fuels and releasing less amount of greenhouse gas. Thermal Energy Storage (TES) systems are developed to store energy efficiently based on the heating or cooling of a specific medium. TES systems can be used in many different applications for heating/ cooling purposes including heating, ventilation, and air conditioning (HVAC) systems, solar power plants for storing thermal energy during the day and release it during the night, and residential buildings for waste heat recovery purposes. In terms of storing thermal energy, TES systems are divided into three major groups including: (i) sensible heat thermal energy storage (SHTES) system (ii) latent heat thermal energy storage (LHTES) system and (iii) thermo-chemical energy storage (TCES) system. However, LHTES systems have attracted more attention of researchers and energy suppliers due to the higher density of thermal energy storage and lower variation in operating temperature [1]. LHTES system is based on heating or cooling a phase change material (PCM) where the thermal energy is absorbed as a latent heat due to the melting process and is released due to solidification process. According to the operation temperature of TES systems, a PCM with proper melting temperature (i.e. phase change temperature) should be used. However, thermal conductivity $(k)$, stability and the amount of latent heat $\left(h_{f g}\right)$ are the other characteristics of selecting a proper PCM [2]. In addition, according to the application of a TES system and the available space for installing this system, different types of geometries such as rectangular, cylindrical, and spherical can be used. Cylindrical TES systems offer the advantages of ease of manufacturing and can be installed both vertically and horizontally. Therefore, in the present investigation, the focus is to study the detailed melting (charging) process of a PCM filled cylindrical TES system. By surveying the literature, many research works can be identified regarding the use of cylindrical TES system filled with PCM. 
Starting with the vertical cylindrical TES system configuration, a numerical investigation of the natural convection through a cylindrical capsule filled with a PCM is conducted by Wu and Lacroix [3]. In their study, the capsule is isothermally heated from the bottom, the top, and the sides. The stream function, vorticity, and temperature are considered in the governing equations and a method called body-fitted coordinates is used to track the irregular shape of solid-liquid phase front. It is reported that conduction is the dominant form of the heat transfer when it is heated from the top. In addition, as the melting process progresses, Nusselt number at the top surface decreases to zero which shows that conduction is dominated during the melting process. Moreover, the bottom surface had the highest rate of heat transfer where the Benard convective cells are dominated. Jones et al. [4], experimentally and numerically studied the melting process of a subcooled paraffin wax. The vertical cylindrical enclosure was isothermally heated from the sides using a hot constant temperature bath, insulated at the top, and a constant temperature condition was applied to the bottom. During the experiments, measurement of temperatures in different radial locations and capturing the melt front locations were performed. By using digital image processing technique, a method for estimating the location of the solid-liquid interface was developed. It is shown that during the melting process of PCM, pure conduction, mixed convection and conduction, convection and shrinking solid are the main heat transfer regimes. A numerical investigation of the melting process of RT-27 was conducted by Shmueli et al. [5]. In their investigation, the cylindrical model was heated isothermally from its sides, insulated from the bottom, and kept open from the top. The model was based on the enthalpy-porosity method and the effects of parameters representing mushy zone and pressure-velocity were considered. Moreover, quantitative information representing the rate of heat transfer and melting fraction was achieved by using image processing of previous experimental work. It is concluded that conduction is the main form of heat transfer at the starting of melting process. As time progresses, natural convection became the dominant form of heat transfer which changes the solid shape of PCM to a conical one. Wang et al. [6], conducted a numerical investigation on the melting process of a PCM with the aim of developing heat transfer correlations. In their study, inward and outward melting process inside a vertical cylindrical enclosure was considered and the model was compared to the previous works. A general agreement was achieved; however, there were some discrepancies due to the influence of heat losses. In addition, the transient heat transfer correlations and total stored energy for a variety of Rayleigh numbers were proposed.

Regarding horizontal cylindrical TES system, Regin et al. [7], performed a numerical and experimental study of the melting of a PCM inside a solar TES system. In their work, horizontal pipes were filled with paraffin wax and subjected to hot water from the surrounding. A numerical model based on enthalpy method was developed and results were compared to experimental investigation. It is reported that Stefan number, radius of the pipe, and range of the phase change temperature are the main dominant parameters, which control the melting process. In addition, by considering the phase change temperature and natural convection in the liquid phase, the agreement between numerical and experimental work was improved. A numerical investigation of the solid-liquid phase change interface for a horizontal cylindrical enclosure was carried out by Mahdaoui et al. [8]. In their work, constant wall temperature and constant heat flux were two main boundary conditions that were investigated. In order to solve the natural convection in the change of the phase, a numerical code based on finite element and enthalpy porosity method was used and the results were validated. It is concluded that at the beginning of melting process, conduction is the dominant form of heat transfer and the local Nusselt number is degraded as time advanced. However, as the melting process progresses, natural convection was developed which significantly affected the shape of the solid-liquid interface. Hosseini et al. [9], conducted an experimental and numerical investigation to study the heat transfer and thermal behaviour of a PCM during constrained condition filled in a shell and tube heat exchanger. Paraffin RT-50 was used as the PCM and several experiments were performed to consider the influence of the inlet temperature of working fluid on melting/solidification process. A numerical model based on an iteration and finite-volume method was developed to study the variation of the molten front with time. Their results showed that rate of heat transfer and charging (melting) time depend on the inlet temperature of working fluid, which by increasing the inlet temperature from $70^{\circ} \mathrm{C}$ to $80^{\circ} \mathrm{C}$, the melting time was degraded by $37 \%$. Moreover, there was a $7.3 \%$ increase in theoretical efficiency of the heat exchanger during the melting process.

According to the literature, the melting process of PCM inside a vertical cylinder insulated from the bottom, isothermally heated from sides, and kept open from the top has not been extensively investigated both experimentally and numerically. In this work, an experimental and numerical study is performed to investigate the melting process of RT-35 as a PCM filled a vertical cylindrical TES system. The lateral walls of the proposed TES system is 
subjected to an isothermal condition with the temperature of $45^{\circ} \mathrm{C}$, while it is insulated at the bottom, and kept open from the top. To perform the investigation, an experimental setup and numerical model were developed to visualize and study the melting process of PCM. This work represents an early stage to study the possible improvements in the melting process by adding nanoparticles into pure PCM.

\section{Problem description}

\subsection{Experimental work}

In this section, a detailed description regarding vertical cylindrical TES system setup and experimental procedures are presented. To visualize the melting process of RT-35 as the PCM, a vertical cylindrical TES prototype is built. The setup consists of an acrylic pipe with an inner diameter of $4.4 \mathrm{~cm}$ and the height of $30 \mathrm{~cm}$ attached to a plastic plate vertically by using a transparent acrylic cement (manufacturer: Scigrip). The bottom part of the enclosure is insulated while it is maintained open from the top to avoid any pressure build on the top of the PCM during the melting process. Then, the cylindrical enclosure is filled with RT-35 (manufacturer: Rubitherm) with the melting temperature $\left(T_{m}\right)$ in the range of $34^{\circ} \mathrm{C}-36^{\circ} \mathrm{C}$ at a specific height $(H)$ in the TES system which represents Rayleigh number $(R a)$ of $10^{7}$ (i.e. the height of the PCM in the TES system is 8.3 $\mathrm{cm})$. Subsequently, the setup was maintained at the room temperature (i.e., $T_{0}=23^{\circ} \mathrm{C}$ ) to initiate the solidification process gradually. Solidification in temperatures close to $T_{m}$ and at a longer time provides the advantage of having less trapped air inside the TES system, leading to having fewer holes inside solid part of the PCM that happens due to the shrinkage of PCM. This offers a more uniform solidliquid interface during melting process and leads to having a fair comparison with numerical results. Next, a transparent water tank equipped with a circulation pump and temperature regulator was used to provide isothermal boundary condition. The temperature of the water inside the tank is set to $45^{\circ} \mathrm{C}$ (i.e. $10^{\circ} \mathrm{C}$ above the $T_{m}$ ) and suddenly the cylindrical TES system is placed inside the hot bath to initiate the melting process. Moreover, a digital camera is used to capture the solid/liquid interface of the PCM periodically. A schematic of the experimental setup can be seen in Figure 1.

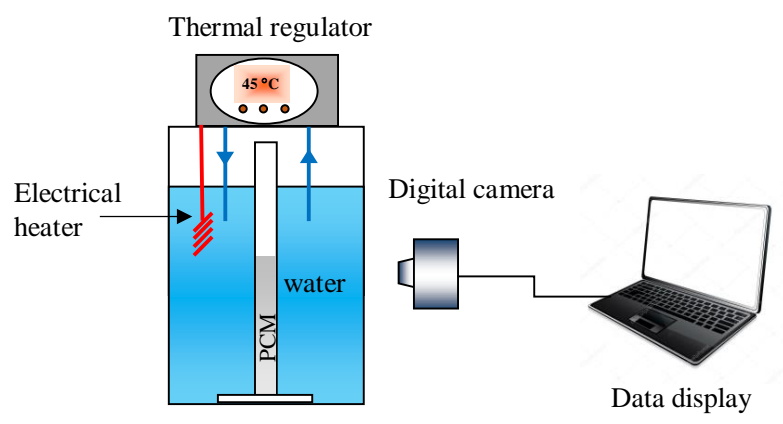

Figure 1: A schematic of the experimental setup

\subsection{Numerical model}

To investigate the flow and thermal fields, a numerical model based on the finite element approach using COMSOL multiphysics is developed. To do so, a 2- $D$ axisymmetric model representing the cylindrical TES system having the same dimensions of the experimental TES system (i.e. inner diameter of the pipe $4.4 \mathrm{~cm}$ and the height of $30 \mathrm{~cm}$ ) is developed. The model is insulated from the bottom and kept open from the top and it is assumed to be filled with RT-35 at a height of $H$ to represent the experimental conditions. The initial temperature of PCM is assumed as $T_{0}$, which is equal to room temperature and lower than $T_{m}$. The model is isothermally heated from the sides at a temperature of $T_{h}$, which is $10^{\circ} \mathrm{C}$ above the $T_{m}$ to initiate the melting process. As the melting process progresses, the liquid-solid interface appears. In this simulation,

the liquid phase of PCM is considered as Newtonian incompressible fluid. In addition, the thermo-physical properties of RT-35 is collected from the literature and the manufacturer. These properties are shown in Table 1.

Table 1: Thermo-physical properties of RT-35 $[10,11]$

\begin{tabular}{lc}
\hline \multicolumn{1}{c}{ Thermo-physical properties } & Value \\
\hline$\rho_{s}\left(\mathrm{~kg} / \mathrm{m}^{3}\right)$ - Density at solid state & 880 \\
$\rho_{l}\left(\mathrm{~kg} / \mathrm{m}^{3}\right)$ - Density at liquid state & 760 \\
$K(\mathrm{~W} / \mathrm{m} \cdot \mathrm{K})$ - Thermal conductivity & 0.2 \\
$\mathrm{C} p(\mathrm{~J} / \mathrm{kg} \cdot \mathrm{K})$ - Specific heat & 2000 \\
$\beta(1 / \mathrm{K})-$ Coefficient of thermal expansion & 0.0006 \\
$h_{f g}(\mathrm{~kJ} / \mathrm{kg})$ - Latent heat of fusion & 160 \\
$\mu(\mathrm{kg} / \mathrm{m} \cdot \mathrm{s})$ - Viscosity & 0.023 \\
$T_{m}\left({ }^{\circ} \mathrm{C}\right)-$ Melting area & 35 \\
Volume expansion $(\%)$ & 12.5 \\
\hline
\end{tabular}


The governing equations representing the conservation of mass, momentum and transferred energy are presented below [12]

$$
\frac{\partial u}{\partial r}+\frac{u}{r}+\frac{\partial w}{\partial z}=0
$$

where $u$ and $w$ are the components of velocity in $r$ direction and $z$ - direction, respectively.

Energy equation:

$$
\frac{\partial T}{\partial t}+u \frac{\partial T}{\partial r}+w \frac{\partial T}{\partial z}=\frac{k}{\rho C_{p}}\left[\frac{1}{r} \frac{\partial}{\partial r}\left(r \frac{\partial T}{\partial r}\right)+\frac{\partial^{2} T}{\partial z^{2}}\right],
$$

where $t, k, \rho$, and $C_{p}$ represent time, thermal conductivity, density, and the specific heat at constant pressure, respectively and the

$$
\begin{aligned}
\frac{\partial w}{\partial t}+u \frac{\partial w}{\partial r}+w \frac{\partial w}{\partial z}= & -\frac{1}{\rho} \frac{\partial p}{\partial z}+\frac{\mu}{\rho}\left(\frac{\partial^{2} w}{\partial r^{2}}+\frac{1}{r} \frac{\partial w}{\partial r}+\frac{\partial^{2} w}{\partial z^{2}}\right) \\
& +g \beta\left(T-T_{m}\right),
\end{aligned}
$$

$r$-momentum equation:

$$
\begin{aligned}
\frac{\partial u}{\partial t}+u \frac{\partial u}{\partial r}+w \frac{\partial u}{\partial z}= & -\frac{1}{\rho} \frac{\partial p}{\partial r} \\
& +\frac{\mu}{\rho}\left(\frac{\partial^{2} u}{\partial r^{2}}+\frac{1}{r} \frac{\partial u}{\partial r}-\frac{u}{r^{2}}+\frac{\partial^{2} u}{\partial z^{2}}\right),
\end{aligned}
$$

where $\mu, g, \beta$, and $T$ represent viscosity, gravitational acceleration and coefficient of thermal expansion and temperature, respectively. For better understanding, it is worthy to read references [13-15] that present the concept of fluid flow in fractal media and its solution techniques.

\subsection{Boundary conditions}

At the beginning of the simulation $(t=0)$, the cylindrical TES system is filled with a certain height of PCM (i.e. $z=$ $H$ ) having a temperature of $T_{0}$ which is lower than $T_{m}$. The bottom wall (i.e. $z=0$ ) is assumed thermally insulated. In order to initiate the melting process, the lateral walls of the cylindrical TES system are subjected to $T_{h}$, which is $10^{\circ} \mathrm{C}$ higher than $T_{m}$. A schematic of the numerical model is presented in Figure 2.

\subsection{Mesh independency test}

Three grid sizes are tested to examine the grid dependency test. The tested grid sizes are fine mesh, number of elements $=6965$, simulation time $=50$ minutes; finer mesh, number of elements $=15062$, simulation time $=1$

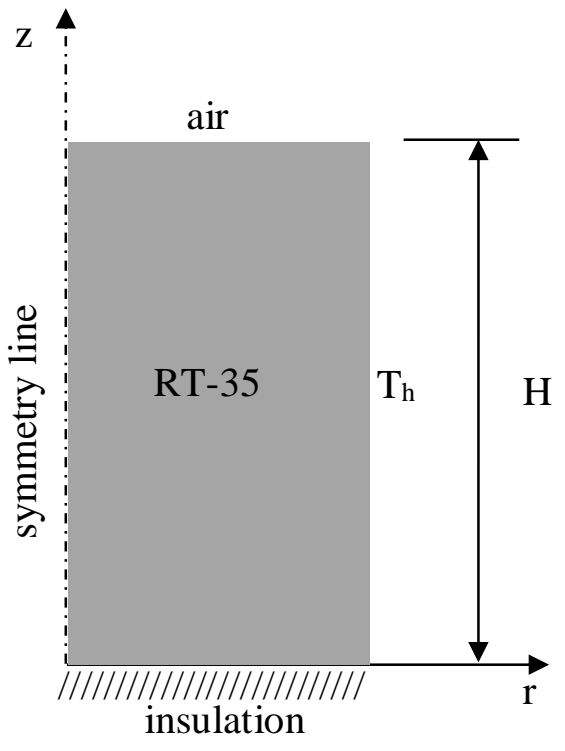

Figure 2: A schematic of the numerical model

hour and 38 minutes, and extra fine mesh, number of elements $=33905$, simulation time $=3$ hours and 47 minutes. The melting fraction is numerically calculated for different mesh sizes. Figure 3 shows that using finer mesh and extra fine mesh lead to almost identical results. However, extra fine mesh takes a longer time to complete the simulation. Therefore, considering the time and the accuracy of the simulation, the finer mesh is considered in the present study.



Figure 3: Melting fraction for the different mesh qualities 


\section{Results and discussion}

Figure 4 shows, experimentally and numerically, the evolution of the melting process of RT-35. The melting process is tracked from time $=300 \mathrm{sec}$. to $7500 \mathrm{sec}$. The RT-35 starts melting after applying a heat source at constant temperature at the walls of the cylinder. The interface shortly appears after initiating the melting process. At 300 sec., the interface is parallel to the walls of the cylinder. The parallel interface indicates that the dominant mode of heat transfer is conduction. Between 1000 and $1800 \mathrm{sec}$, the melted RT-35 occupies more space at the upper part of the cylinder. With ongoing heating, the interface deforms, and the remaining solid RT-35 takes the shape of a cone starting from $3900 \mathrm{sec}$. to the end of the melting process. Most of the melted RT-35 occupies the upper part of the cylinder, while less amount of solid RT-35 is available at the lower part of the cylinder. The warm liquid RT-35 that has been heated through the walls of the cylinder rises up as it becomes lighter. The warm melted RT-35 accumulates at the top of the cylinder due to the thermal stratification effect. Since the melted RT-35 cannot cross the impermeable surface, the colder melted RT-35 moves downwards to give space for the warmer melted RT-35. As a result, the transferred thermal energy to the solid RT-35 gradually drops as the melted RT-35 moves downwards. As it can be seen from Figure 4, the computed numerical results are in a good agreement with the conducted experiment results.

Figure 5 shows the comparison of melting fraction with time between the experimental and numerical results. The melting fraction $(M F)$ is calculated by using Eq. 5.

\section{$M F=\frac{\text { Volume of the liquid PCM }}{\text { Total volume of the PCM }(\text { solid }+ \text { liquid })} \times 100$}

In order to calculate the melting fraction for the results obtained by digital photos from experimental work, the image processing method has been used. An almost linear trend is observed between the melting fraction and time in both results with a higher melting at the beginning followed by a slower melting rate as melting progresses. At the beginning of the melting process, the temperature difference between the PCM in the TES system and the constant temperature bath is the highest and therefore, melting rate of PCM is highest. As the melting process advances, the amount of liquid PCM in the TES system increases and so does its temperature. As a result, the temperature difference between the PCM in the TES system
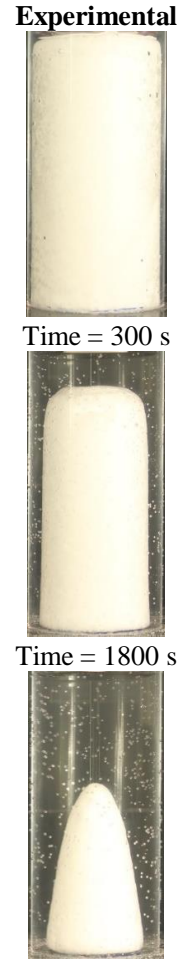

Time $=4800$

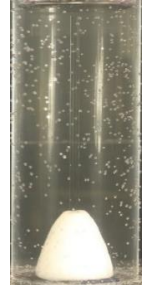

Time $=6800 \mathrm{~s}$
Time $=1800$

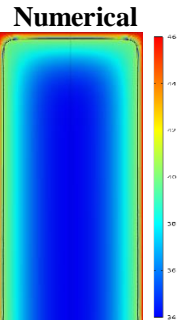

Time $=300 \mathrm{~s}$
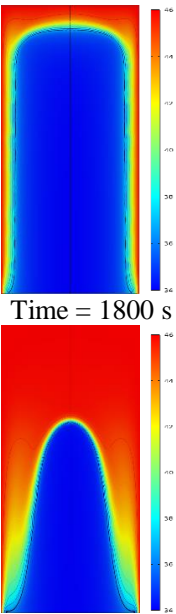

Time $=4800$

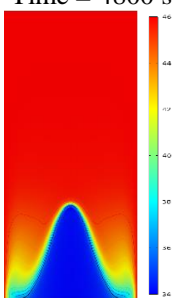

Time $=6800 \mathrm{~s}$

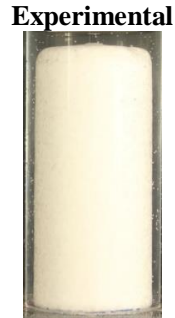

Time $=1000 \mathrm{~s}$

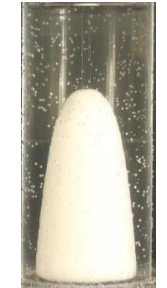

Time $=3900$

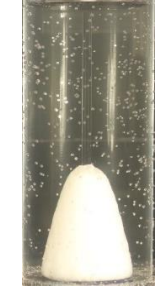

Time $=5800$

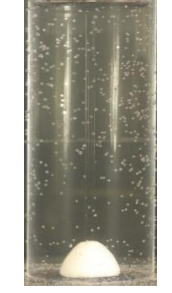

Time $=7500 \mathrm{~s}$

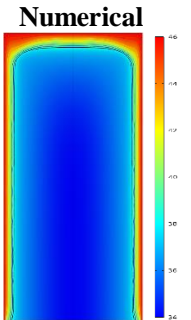

Time $=1000 \mathrm{~s}$

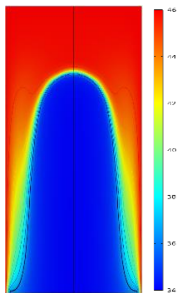

Time $=3900 \mathrm{~s}$

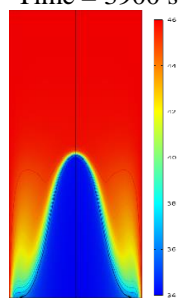

Time $=5800$

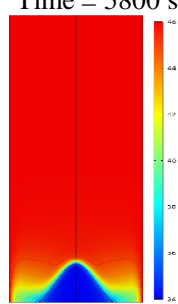

Time $=7500$
Figure 4: Comparison of melting pattern between the experimental and numerical results

and the constant temperature bath decreases and the melting rate of PCM decreases.

As it can be seen from Figure 5, there are some discrepancies in the value of melting fraction obtained by numerical and experimental work. The main reasons of these differences could be (i) ignoring the thermal resistance of acrylic pipe in the numerical model; (ii) improper insulation at the bottom of cylindrical TES system in the experimental work and (iii) ignoring the thermal expansion of the PCM during the melting process in the numerical model.

Figure 6 shows the amount of transferred heat flux into the TES with time. At the beginning of the melting process, the PCM which is attached to the TES wall is solid and at a lower temperature than the bath temperature. At this point, the temperature difference between the cold PCM and hot lateral walls is high which causes high heat trans- 


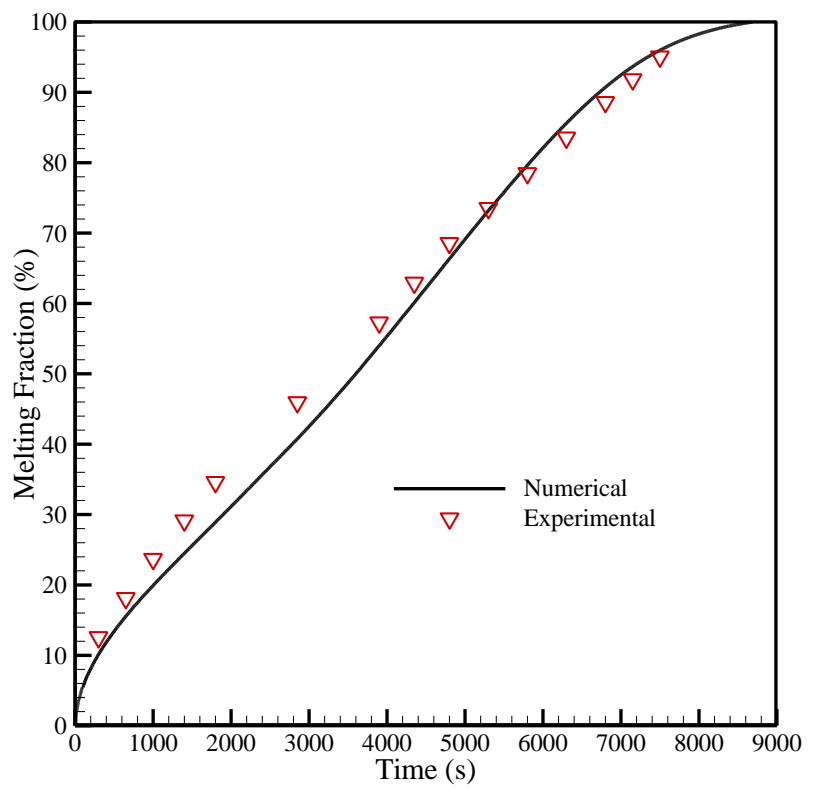

Figure 5: A comparison of melting fraction with time between the experimental and numerical results

fer into the TES. With ongoing heating, the PCM close to the wall becomes warmer which reduces the temperature difference between inside and outside the TES. As a result, the heat transfer drops with time.

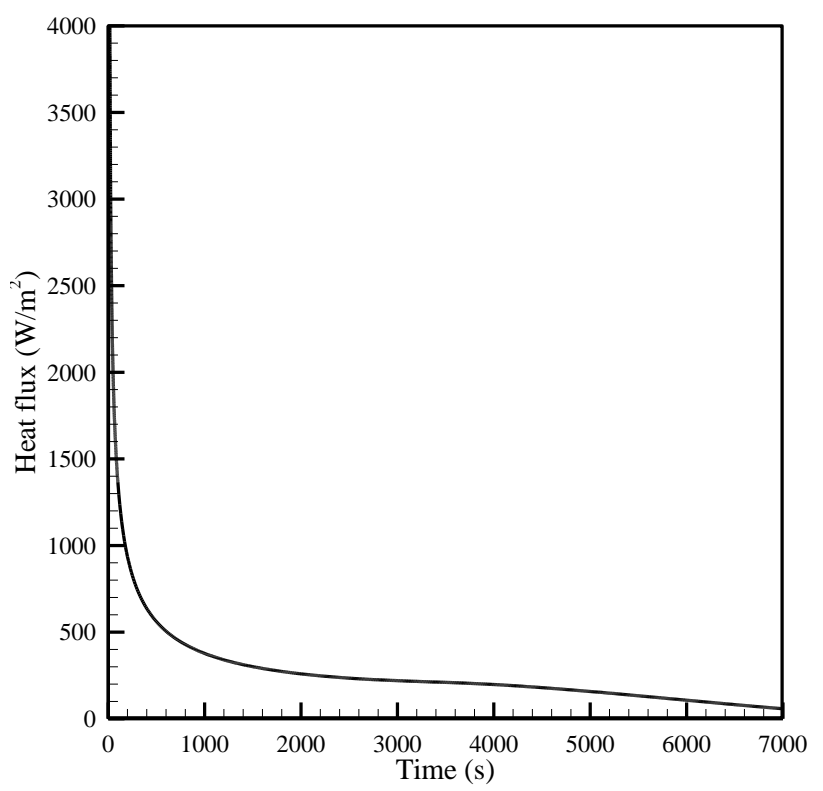

Figure 6: The heat flux transferred into the TES

Figure 7 shows the trend of surface averaged Nusselt number $(\mathrm{Nu}), \mathrm{Nu}$ throughout the melting of RT-35. The heat transfer rate at the beginning of the melting process is high

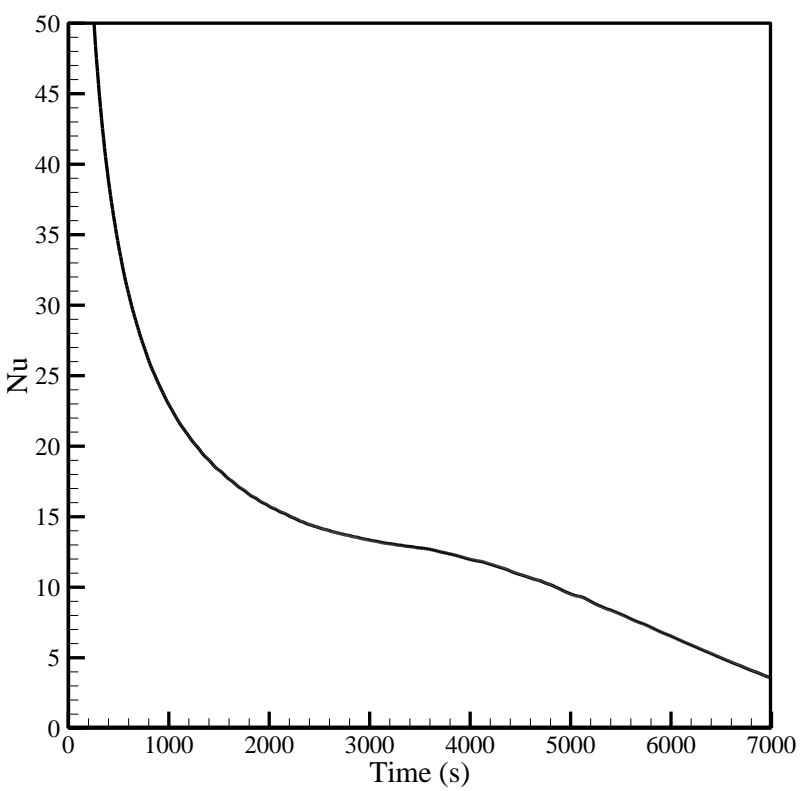

Figure 7: Nusselt number behavior throughout the melting process

due to the high difference temperature between the hot walls of the cylinder and the cold solid RT-35. At the beginning of melting process, the magnitude of $\mathrm{Nu}$ number is high due to a large temperature gradient. From the initial stages onward, the magnitude of $\mathrm{Nu}$ number drops with time until it reaches it is minimum value as the thermal equilibrium condition is achieved.

\section{Conclusion}

A numerical and experimental investigation on the melting process of RT-35 filling a vertical cylindrical enclosure is conducted. The numerical model results are compared with the experimental work and good agreement is achieved. However, some discrepancy is observed. It is concluded that at the early stage of melting process, conduction is the dominant form of heat transfer from the walls. As the melting process progresses, natural convection is developed until it dominates the heat transfer process. At the early stages of the process, the heat transfer rate is high which is degraded at the later stages due to the decrease in temperature gradient between cold PCM and hot lateral walls of cylindrical TES system.

\section{Nomenclature}

$c_{p}$ specific heat at constant pressure $[\mathrm{kJ} / \mathrm{kg} \mathrm{K}]$

$H$ height of the filled PCM in TES system [cm]

$h_{f g}$ latent heat of fusion $[\mathrm{kJ} / \mathrm{kg}]$

$g$ gravitational acceleration $\left[\mathrm{m} / \mathrm{s}^{2}\right]$ 
$k$ thermal conductivity $[\mathrm{W} / \mathrm{m} \cdot \mathrm{K}]$

$T$ temperature $\left[{ }^{\circ} \mathrm{C}\right]$

Greek symbols

$\beta$ coefficient of thermal expansion $[1 / \mathrm{K}]$

$\mu$ dynamic viscosity [Pa.s]

$\rho$ density $\left[\mathrm{kg} / \mathrm{m}^{3}\right]$

Subscripts

$h$ hot

$m$ melt

$O$ initial

Abbreviations

TES thermal energy storage

SHTES sensible heat thermal energy storage

LHTES latent heat thermal energy storage

TCES thermos-chemical energy storage

MF melting fraction

PCM phase change material

RT Rubitherm

Ra Rayleigh number

\section{References}

[1] Dhaidan N.S., Khodadadi J.M., Melting and convection of phase change materials in different shape containers: A review, Renew. Sustain. Energy Rev., 2015, 43, 449-477.

[2] Medrano M., Yilmaz M.O., Nougues M., Martorell I., Roca J., Cabeza L.F., Experimental evaluation of commercial heat exchangers for use as PCM thermal storage systems, Appl. Energy, 2009, 86, 2046-2055.

[3] Wu Y.K., Lacroix M., Melting of aPCM inside a vertical cylindrical capsule, Int. J. Numer. Methods in Fluids, 1995, 20, 559-572.
[4] Jones B.J., Sun D., Krishnan S., Garimella S.V., Experimental and numerical study of melting in a cylinder, Int. J. Heat Mass Transfer, 2006, 49, 2724-2738.

[5] Shmueli H., Ziskind G., Letan R., Melting in a vertical cylindrical tube: numerical investigation and comparison with experiments, Int. J. Heat Mass Transfer, 2010, 53, 4082-4091.

[6] Wang S., Faghri A., Bergman T. L., Melting in cylindrical enclosures: numerical modeling and heat transfer correlations, $\mathrm{Nu}$ mer. Heat Transfer Part A: Applications, 2012, 61, 837-859.

[7] Regin A.F., Solanki S.C., Saini J.S., Latent heat thermal energy storage using cylindrical capsule: Numerical and experimental investigations, Renew. Energy, 2006, 31, 2025-2041.

[8] Mahdaoui M., Kousksou T., Blancher S., Ait Msaad A., El Rhafiki T., Mouqallid M., A numerical analysis of solid-liquid phase change heat transfer around a horizontal cylinder, Appl. Math. Modelling, 2014, 38, 1101-1110.

[9] Hosseini M. J., Rahimi M., Bahrampoury R., Experimental and computational evolution of a shell and tube heat exchanger as a PCM thermal storage system, Int. J. Heat Mass Transfer, 2014, $50,128-136$.

[10] www.rubitherm.eu

[11] Rahimi M., Ranjbar A.A., Ganji D.D., Sedighi K., Hosseini M. J., Bahrampoury R., Analysis of geometrical and operational parameters of PCM in a fin and tube heat exchanger, Int. Comm. Heat Transfer, 2014, 53, 109-115.

[12] Bejan A., Conv. Heat Transfer., 2013, New York, Wiley

[13] Yang X., Baleanu D., Tenreiro Machado J.A., Systems of NavierStokes equations on Cantor sets, Math. Probl. Eng., 2013, 1-8.

[14] Yang X., Srivastava H.M., An asymptotic perturbation solution for a linear oscillator of free damped vibrations in fractal medium described by local fractional derivatives, Comm. Nonlinear Sci. Numer. Simul., 2015, 29, 499-504.

[15] Yang X., Zhang Z., Srivastava H. M., Some new applications for heat and fluid flows via fractional derivatives without singular kernel, Thermal Sci., 2016, 20, 833-839. 